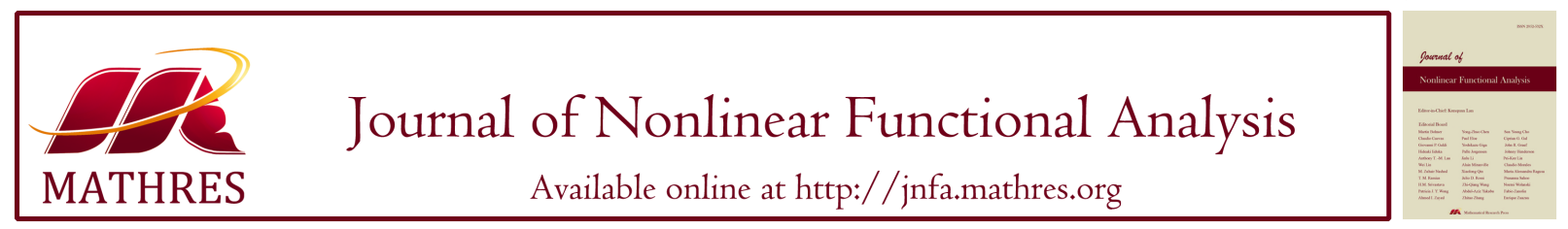

\title{
EXISTENCE RESULTS AND THE DIMENSION OF THE SOLUTION SET FOR A NONLOCAL INCLUSIONS PROBLEM WITH MIXED FRACTIONAL DERIVATIVES AND INTEGRALS
}

\author{
AHMED ALSAEDI ${ }^{1}$, ABRAR BROOM $^{1}$, SOTIRIS K. NTOUYAS ${ }^{2,1, *}$, BASHIR AHMAD ${ }^{1}$ \\ ${ }^{1}$ Nonlinear Analysis and Applied Mathematics (NAAM)-Research Group, Department of Mathematics, \\ Faculty of Science, King Abdulaziz University, Jeddah 21589, Saudi Arabia \\ ${ }^{2}$ Department of Mathematics, University of Ioannina, 45110 Ioannina, Greece
}

\begin{abstract}
We prove the existence of solutions for fractional differential inclusions containing rightCaputo and left-Riemann-Liouville fractional derivatives of different orders and right-left RiemannLiouville fractional integrals, supplemented with nonlocal boundary conditions. Our first existence result dealing with the convex valued maps involved in the given problem is derived by applying the nonlinear alternative of Leray-schauder type. We apply Wegrzyk's fixed point theorem to prove our second result, which is concerned with the generalized contraction (nonconvex valued) maps. We also discuss the dimension of the solution set.

Keywords. Fractional differential inclusion; Boundary value problem; Fixed point theorems; RiemannLiouville fractional integral; Fractional calculus.
\end{abstract}

\section{INTRODUCTION}

The tools of fractional calculus are found to be of great utility in improving the mathematical modeling of complex dynamical systems. An important characteristic of fractional-order operators is their nonlocal nature that takes into account the past history of the underlying phenomena, such as transport processes [1], economic processes [2], viscoelastic materials [3], etc. During the last few decades, there has been a remarkable development in the area of boundary value problems of fractional differential equations; see, e.g., [4]-[6]. However, nonlocal boundary value problems involving left and right fractional derivatives need further attention. Some recent results on this class of problems can be found in [7,8]. It is worthwhile to mention that

\footnotetext{
${ }^{*}$ Corresponding author.

E-mail addresses: aalsaedi@hotmail.com (A. Alsaedi), abrarbroom1992@gmail.com (A. Broom), sntouyas@ uoi.gr (S.K. Ntouyas), bashirahmad_qau@yahoo.com (B. Ahmad).

Received April 4, 2020; Accepted June 28, 2020.
} 
the one-sided derivatives and their combinations appear in the study of Euler-Lagrange equations [9], steady heat-transfer in fractal media [10], electromagnetic waves phenomena [11], etc. Multivalued (inclusions) problems serve as an important tool in the investigation of dynamical systems and stochastic processes; see, e.g., [12]-[14]. For the applications in queuing networks, finance, and climate control, one refers to [15]. On the other hand, one can find the theoretical development of the topic in [16]-[18] and the references cited therein.

In this paper, we discuss the existence of solutions for a nonlocal fractional differential inclusions problem involving right-Caputo and left-Riemann-Liouville fractional derivatives of different orders and right-left Riemann-Liouville fractional integrals of the form:

$$
\left\{\begin{array}{l}
{ }^{C} \mathscr{D}_{1-}^{\rho} R L \mathscr{D}_{0+}^{\beta} y(t) \in F(t, y(t))-\lambda I_{1-}^{p} I_{0+}^{q} G(t, y(t)), t \in[0,1] \\
y(0)=y(\xi)=0, \quad y(1)=\delta y(\mu), 0<\xi<\mu<1
\end{array}\right.
$$

where ${ }^{C} \mathscr{D}_{1-}^{\rho}$ and ${ }^{R L} \mathscr{D}_{0+}^{\beta}$ respectively denote the right Caputo and left Riemann-Liouville fractional derivatives of order $\rho \in(1,2]$ and $\beta \in(0,1], I_{1-}^{p}$ and $I_{0+}^{q}$ denote the right and left Riemann-Liouville fractional integrals of orders $p, q>0$ respectively, $F, G:[0,1] \times \mathbb{R} \rightarrow \mathscr{P}(\mathbb{R})$ are the given multivalued maps, and $\mathscr{P}(\mathbb{R})$ is the family of all nonempty subsets of $\mathbb{R}$.

Motivated by the recent results on fractional multivalued problems, we consider the inclusions case of the problem studied in [19] in this paper and explore the sufficient criteria ensuring the existence of solutions for the inclusions problem (1.1) when $F$ and $G$ are convex as well as nonconvex multivalued maps. In case of nonconvex multivalued maps $F$ and $G$, we apply a generalized version of Covits and Nadler's fixed point theorem [20] due to Wegrzyk [21], which deals with the generalized contractions. We also discuss the dimension of the solution set for problem (1.1). The rest of the paper is arranged as follows. Section 2 contains the background material related to our study. In Section 3, we present our main existence results for problem (1.1), while the dimension of the solution set for the given problem is discussed in Section 4.

\section{PRELIMINARIES}

Let us first recall the basic definitions related to our work.

Definition 2.1. [22] The left and right Riemann-Liouville fractional integrals of order $\alpha>0$ for $x \in L_{1}[a, b]$, existing almost everywhere on $[a, b]$, are respectively defined by

$$
I_{a+}^{\alpha} x(t)=\int_{a}^{t} \frac{(t-s)^{\alpha-1}}{\Gamma(\alpha)} x(s) d s \text { and } I_{b-}^{\alpha} x(t)=\int_{t}^{b} \frac{(s-t)^{\alpha-1}}{\Gamma(\alpha)} x(s) d s .
$$

Definition 2.2. [22] For $x \in A C^{n}[a, b]$, the left Riemann-Liouville and the right Caputo fractional derivatives of order $\alpha \in(n-1, n], n \in \mathbb{N}$, existing almost everywhere on $[a, b]$, are respectively defined by

$$
{ }^{R L} \mathscr{D}_{a+}^{\alpha} x(t)=\frac{d^{n}}{d t^{n}} \int_{a}^{t} \frac{(t-s)^{n-\delta-1}}{\Gamma(n-\alpha)} x(s) d s \text { and }{ }^{C} \mathscr{D}_{b-}^{\alpha} x(t)=(-1)^{n} \int_{t}^{b} \frac{(s-t)^{n-\alpha-1}}{\Gamma(n-\alpha)} x^{(n)}(s) d s .
$$

The following lemma, concerning a linear variant of problem (1.1), was proved in [19]. 
Lemma 2.3. Let $u, v \in C[0,1] \cap L(0,1)$. Then the linear problem

$$
\left\{\begin{array}{l}
{ }^{C} \mathscr{D}_{1-}^{\rho}{ }^{R L} \mathscr{D}_{0+}^{\beta} y(t)+\lambda I_{1-}^{p} I_{0+}^{q} v(t)=u(t), \quad t \in J:=[0,1], \\
y(0)=y(\xi)=0, \quad y(1)=\delta y(\mu),
\end{array}\right.
$$

is equivalent to the fractional integral equation:

$$
\begin{aligned}
y(t)= & \int_{0}^{t} \frac{(t-s)^{\beta-1}}{\Gamma(\beta)}\left[I_{1-}^{\rho} u(s)-\lambda I_{1-}^{\rho+p} I_{0+}^{q} v(s)\right] d s \\
& +a_{1}(t)\left\{\delta \int_{0}^{\mu} \frac{(\mu-s)^{\beta-1}}{\Gamma(\beta)}\left[I_{1-}^{\rho} u(s)-\lambda I_{1-}^{\rho+p} I_{0+}^{q} v(s)\right] d s\right. \\
& \left.-\int_{0}^{1} \frac{(1-s)^{\beta-1}}{\Gamma(\beta)}\left[I_{1-}^{\rho} u(s)-\lambda I_{1-}^{\rho+p} I_{0+}^{q} v(s)\right] d s\right\} \\
& +a_{2}(t) \int_{0}^{\xi} \frac{(\xi-s)^{\beta-1}}{\Gamma(\beta)}\left[I_{1-}^{\rho} u(s)-\lambda I_{1-}^{\rho+p} I_{0+}^{q} v(s)\right] d s,
\end{aligned}
$$

where

$$
a_{1}(t)=\frac{1}{\Lambda}\left[\xi^{\beta+1} t^{\beta}-\xi^{\beta} t^{\beta+1}\right], a_{2}(t)=\frac{1}{\Lambda}\left[t^{\beta}\left(1-\delta \mu^{\beta+1}\right)-t^{\beta+1}\left(1-\delta \mu^{\beta}\right)\right],
$$

and it is assumed that

$$
\Lambda=\xi^{\beta+1}\left(1-\delta \mu^{\beta}\right)-\xi^{\beta}\left(1-\delta \mu^{\beta+1}\right) \neq 0
$$

\section{Existence RESUlts}

Let $C[0,1]$ denote the Banach space of continuous functions from $[0,1]$ into $\mathbb{R}$ with the norm $\|x\|_{\infty}=\sup _{t \in[0,1]}|x(t)|$. Let $L^{1}([0,1], \mathbb{R})$ be the Banach space of measurable functions $x:[0,1] \rightarrow$ $\mathbb{R}$, which are Lebesgue integrable and normed by $\|x\|_{L^{1}}=\int_{0}^{1}|x(t)| d t$. For some related concepts about multi-valued maps, we refer the reader to [5, 23, 24].

For each $y \in C([0,1], \mathbb{R})$, one defines the sets of selections of $F$ and $G$ by

$$
\begin{aligned}
& S_{F, y}:=\left\{f \in L^{1}([0,1], \mathbb{R}): f(t) \in F(t, y)\right\}, \\
& \widehat{S}_{G, y}:=\left\{g \in L^{1}([0,1], \mathbb{R}): g(t) \in G(t, y)\right\} .
\end{aligned}
$$

Definition 3.1. A function $y \in C([0,1], \mathbb{R})$ is a solution of boundary value problem (1.1) if $y(0)=y(\xi)=0, y(1)=\delta y(\mu)$, and there exist functions $f \in S_{F, y}, g \in \widehat{S}_{G, y}$ such that $f(t) \in$ $F(t, y(t)), g(t) \in G(t, y(t))$ and

$$
\begin{aligned}
y(t)= & \int_{0}^{t} \frac{(t-s)^{\beta-1}}{\Gamma(\beta)}\left[I_{1-}^{\rho} f(s)-\lambda I_{1-}^{\rho+p} I_{0+}^{q} g(s)\right] d s \\
& +a_{1}(t)\left\{\delta \int_{0}^{\mu} \frac{(\mu-s)^{\beta-1}}{\Gamma(\beta)}\left[I_{1-}^{\rho} f(s)-\lambda I_{1-}^{\rho+p} I_{0+}^{q} g(s)\right] d s\right. \\
& \left.-\int_{0}^{1} \frac{(1-s)^{\beta-1}}{\Gamma(\beta)}\left[I_{1-}^{\rho} f(s)-\lambda I_{1-}^{\rho+p} I_{0+}^{q} g(s)\right] d s\right\} \\
& +a_{2}(t) \int_{0}^{\xi} \frac{(\xi-s)^{\beta-1}}{\Gamma(\beta)}\left[I_{1-}^{\rho} f(s)-\lambda I_{1-}^{\rho+p} I_{0+}^{q} g(s)\right] d s .
\end{aligned}
$$


3.1. The upper semicontinuous case (Convex valued). In this subsection, we prove an existence result for problem (1.1) when both $F$ and $G$ are convex multivalued maps by applying the nonlinear alternative of Leray-schauder type [25].

Theorem 3.2. Assume that:

$\left(\mathbf{H}_{1}\right) F, G:[0,1] \times \mathbb{R} \rightarrow \mathscr{P}_{c p, c}(\mathbb{R})$ are $L^{1}$-Carathéodory, where $\mathscr{P}_{c p, c}(\mathbb{R})=\{\mathscr{U} \in \mathscr{P}(\mathbb{R}): \mathscr{U}$ is compact and convex $\}$;

$\left(\mathbf{H}_{2}\right)$ there exist functions $\sigma_{1}, \sigma_{2} \in L^{1}\left([0,1], \mathbb{R}^{+}\right)$, and continuous nondecreasing functions $\theta_{1}, \theta_{2}: \mathbb{R}^{+} \rightarrow \mathbb{R}^{+}$such that

$$
\begin{aligned}
& \|F(t, y)\|=\sup \{|f|: f(t) \in F(t, y)\} \leq \sigma_{1}(t) \theta_{1}(|y|), \\
& \|G(t, y)\|=\sup \{|g|: g(t) \in G(t, y)\} \leq \sigma_{2}(t) \theta_{2}(|y|),
\end{aligned}
$$

for each $(t, y) \in[0,1] \times \mathbb{R}$

$\left(\mathbf{H}_{3}\right)$ there exists a constant $M>0$ such that

$$
\frac{\Gamma(\rho) \Gamma(\rho+p) \Gamma(q) M}{\left(\Gamma(\rho+p) \Gamma(q) \theta_{1}(M)\left\|\sigma_{1}\right\|_{L^{1}}+|\lambda| \Gamma(\rho) \theta_{2}(M)\left\|\sigma_{2}\right\|_{L^{1}}\right) \tilde{\Delta}}>1
$$

where

$$
\tilde{\Delta}=\frac{1}{\Gamma(\beta+1)}\left[1+\bar{a}_{1}(|\delta|+1)+\bar{a}_{2}\right], \bar{a}_{1}=\max _{t \in[0,1]}\left|a_{1}(t)\right|, \bar{a}_{2}=\max _{t \in[0,1]}\left|a_{2}(t)\right| .
$$

Then boundary value problem (1.1) has at least one solution on [0,1].

Proof. In order to transform problem (1.1) into a fixed point problem, let us consider the multivalued map $\Omega: C([0,1], \mathbb{R}) \rightarrow \mathscr{P}(C([0,1], \mathbb{R}))$ as

$$
\begin{aligned}
\Omega(y)=\{h \in C([0,1], \mathbb{R}): \quad & h(t)=\int_{0}^{t} \frac{(t-s)^{\beta-1}}{\Gamma(\beta)}\left[I_{1-}^{\rho} f(s)-\lambda I_{1-}^{\rho+p} I_{0+}^{q} g(s)\right] d s \\
& +a_{1}(t)\left[\delta \int_{0}^{\mu} \frac{(\mu-s)^{\beta-1}}{\Gamma(\beta)}\left[I_{1-}^{\rho} f(s)-\lambda I_{1-}^{\rho+p} I_{0+}^{q} g(s)\right] d s\right. \\
& \left.-\int_{0}^{1} \frac{(1-s)^{\beta-1}}{\Gamma(\beta)}\left[I_{1-}^{\rho} f(s)-\lambda I_{1-}^{\rho+p} I_{0+}^{q} g(s)\right] d s\right] \\
& \left.+a_{2}(t) \int_{0}^{\xi} \frac{(\xi-s)^{\beta-1}}{\Gamma(\beta)}\left[I_{1-}^{\rho} f(s)-\lambda I_{1-}^{\rho+p} I_{0+}^{q} g(s)\right] d s\right\},(3.2
\end{aligned}
$$

for $f \in S_{F, y}, g \in \widehat{S}_{G, y}$.

We next show that $\Omega$ satisfies all the assumptions of nonlinear alternative of Leray-Schauder type [25] in several steps.

Step 1. $(\Omega(y)$ is convex for each $y \in C([0,1], \mathbb{R}))$. 
Let $h_{1}, h_{2} \in \Omega(y)$. Then there exist $f_{1}, f_{2} \in S_{F, y}, g_{1}, g_{2} \in \widehat{S}_{G, y}$ such that, for each $t \in[0,1]$,

$$
\begin{aligned}
h_{i}(t)= & \int_{0}^{t} \frac{(t-s)^{\beta-1}}{\Gamma(\beta)}\left[I_{1-}^{\rho} f_{i}(s)-\lambda I_{1-}^{\rho+p} I_{0+}^{q} g_{i}(s)\right] d s \\
& +a_{1}(t)\left\{\delta \int_{0}^{\mu} \frac{(\mu-s)^{\beta-1}}{\Gamma(\beta)}\left[I_{1-}^{\rho} f_{i}(s)-\lambda I_{1-}^{\rho+p} I_{0+}^{q} g_{i}(s)\right] d s\right. \\
& \left.-\int_{0}^{1} \frac{(1-s)^{\beta-1}}{\Gamma(\beta)}\left[I_{1-}^{\rho} f_{i}(s)-\lambda I_{1-}^{\rho+p} I_{0+}^{q} g_{i}(s)\right] d s\right\} \\
& +a_{2}(t) \int_{0}^{\xi} \frac{(\xi-s)^{\beta-1}}{\Gamma(\beta)}\left[I_{1-}^{\rho} f_{i}(s)-\lambda I_{1-}^{\rho+p} I_{0+}^{q} g_{i}(s)\right] d s, i=1,2 .
\end{aligned}
$$

For each $t \in[0,1]$ and $0 \leq v \leq 1$, we can find that

$$
\begin{aligned}
& {\left[v h_{1}+(1-v) h_{2}\right](t)} \\
& =\int_{0}^{t} \frac{(t-s)^{\beta-1}}{\Gamma(\beta)}\left[I_{1-}^{\rho}\left[v f_{1}(s)+(1-v) f_{2}(s)\right]-\lambda I_{1-}^{\rho+p} I_{0+}^{q}\left[v g_{1}(s)+(1-v) g_{2}(s)\right]\right] d s \\
& +a_{1}(t)\left\{\delta \int _ { 0 } ^ { \mu } \frac { ( \mu - s ) ^ { \beta - 1 } } { \Gamma ( \beta ) } \left[I_{1-}^{\rho}\left[v f_{1}(s)+(1-v) f_{2}(s)\right]\right.\right. \\
& \left.-\lambda I_{1-}^{\rho+p} I_{0+}^{q}\left[v g_{1}(s)+(1-v) g_{2}(s)\right]\right] d s \\
& \left.-\int_{0}^{1} \frac{(1-s)^{\beta-1}}{\Gamma(\beta)}\left[I_{1-}^{\rho}\left[v f_{1}(s)+(1-v) f_{2}(s)\right]-\lambda I_{1-}^{\rho+p} I_{0+}^{q}\left[v g_{1}(s)+(1-v) g_{2}(s)\right]\right] d s\right\} \\
& +a_{2}(t) \int_{0}^{\xi} \frac{(\xi-s)^{\beta-1}}{\Gamma(\beta)}\left[I_{1-}^{\rho}\left[v f_{1}(s)+(1-v) f_{2}(s)\right]\right. \\
& \left.-\lambda I_{1-}^{\rho+p} I_{0+}^{q}\left[v g_{1}(s)+(1-v) g_{2}(s)\right]\right] d s .
\end{aligned}
$$

Since $S_{F, y}, \widehat{S}_{G, y}$ are convex $\left(F, G\right.$ have convex values), therefore it follows that $v h_{1}+(1-v) h_{2} \in$ $\Omega(y)$.

Step 2. ( $\Omega$ maps bounded sets into bounded sets in $C([0,1], \mathbb{R}))$.

For $r>0$, let $B_{r}=\left\{y \in C([0,1], \mathbb{R}):\|y\|_{\infty} \leq r\right\}$ be a bounded ball in $C([0,1], \mathbb{R})$. Then, for each $h \in \Omega(y), y \in B_{r}$, there exist $f \in S_{F, y}, g \in \widehat{S}_{G, y}$ such that

$$
\begin{aligned}
h(t)= & \int_{0}^{t} \frac{(t-s)^{\beta-1}}{\Gamma(\beta)}\left[I_{1-}^{\rho} f(s)-\lambda I_{1-}^{\rho+p} I_{0+}^{q} g(s)\right] d s \\
& +a_{1}(t)\left[\delta \int_{0}^{\mu} \frac{(\mu-s)^{\beta-1}}{\Gamma(\beta)}\left[I_{1-}^{\rho} f(s)-\lambda I_{1-}^{\rho+p} I_{0+}^{q} g(s)\right] d s\right. \\
& \left.-\int_{0}^{1} \frac{(1-s)^{\beta-1}}{\Gamma(\beta)}\left[I_{1-}^{\rho} f(s)-\lambda I_{1-}^{\rho+p} I_{0+}^{q} g(s)\right] d s\right] \\
& +a_{2}(t) \int_{0}^{\xi} \frac{(\xi-s)^{\beta-1}}{\Gamma(\beta)}\left[I_{1-}^{\rho} f(s)-\lambda I_{1-}^{\rho+p} I_{0+}^{q} g(s)\right] d s .
\end{aligned}
$$


In view of $\left(\mathbf{H}_{2}\right)$, we have

$$
\begin{aligned}
|h(t)|= & \int_{0}^{t} \frac{(t-s)^{\beta-1}}{\Gamma(\beta)}\left[I_{1-}^{\rho}|f(s)|+|\lambda| I_{1-}^{\rho+p} I_{0+}^{q}|g(s)|\right] d s \\
& +\left|a_{1}(t)\right|\left[|\delta| \int_{0}^{\mu} \frac{(\mu-s)^{\beta-1}}{\Gamma(\beta)}\left[I_{1-}^{\rho}|f(s)|+|\lambda| I_{1-}^{\rho+p} I_{0+}^{q}|g(s)|\right] d s\right. \\
& \left.+\int_{0}^{1} \frac{(1-s)^{\beta-1}}{\Gamma(\beta)}\left[I_{1-}^{\rho}|f(s)|+|\lambda| I_{1-}^{\rho+p} I_{0+}^{q}|g(s)|\right] d s\right] \\
& +\left|a_{2}(t)\right| \int_{0}^{\xi} \frac{(\xi-s)^{\beta-1}}{\Gamma(\beta)}\left[I_{1-}^{\rho}|f(s)|+|\lambda| I_{1-}^{\rho+p} I_{0+}^{q}|g(s)|\right] d s \\
\leq & \left(\frac{\theta_{1}(r)}{\Gamma(\rho)} \int_{0}^{1} \sigma_{1}(t) d t+\frac{|\lambda| \theta_{2}(r)}{\Gamma(\rho+p) \Gamma(q)} \int_{0}^{1} \sigma_{2}(t) d t\right) \\
& \times \frac{1}{\Gamma(\beta+1)}\left[1+\bar{a}_{1}(|\delta|+1)+\bar{a}_{2}\right] .
\end{aligned}
$$

Thus

$$
\|h\|_{\infty} \leq\left(\frac{\theta_{1}(r)}{\Gamma(\rho)}\left\|\sigma_{1}\right\|_{L^{1}}+\frac{|\lambda| \theta_{2}(r)}{\Gamma(\rho+p) \Gamma(q)}\left\|\sigma_{2}\right\|_{L^{1}}\right) \tilde{\Delta} .
$$

Indeed, in (3.3), we have used the following estimates $(\rho \in(1,2], \beta \in(0,1], p>0, q>1)$ :

$$
\begin{gathered}
\int_{0}^{t} \frac{(t-s)^{\beta-1}}{\Gamma(\beta)} I_{1-}^{\rho} \sigma_{1}(s) d s \leq \frac{1}{\Gamma(\beta+1) \Gamma(\rho)} \int_{0}^{1} \sigma_{1}(u) d u \\
\int_{0}^{t} \frac{(t-s)^{\beta-1}}{\Gamma(\beta)} I_{1-}^{\rho+p} I_{0+}^{q} \sigma_{2}(s) d s \leq \frac{1}{\Gamma(\beta+1) \Gamma(\rho+p) \Gamma(q)} \int_{0}^{1} \sigma_{2}(t) d t .
\end{gathered}
$$

Step 3. ( $\Omega$ maps bounded sets into equicontinuous sets of $C([0,1], \mathbb{R}))$.

Let $t_{1}, t_{2} \in[0,1]$ with $t_{1}<t_{2}$ and let us take any element $y \in B_{r}, h \in \Omega(y)$. Then there exist $f \in S_{F, y}, g \in \widehat{S}_{G, y}$ such that, for each $t \in[0,1]$,

$$
\begin{aligned}
\left|h\left(t_{2}\right)-h\left(t_{1}\right)\right|= & \mid \int_{0}^{t_{1}} \frac{\left|\left(t_{2}-s\right)^{\beta-1}-\left(t_{1}-s\right)^{\beta-1}\right|}{\Gamma(\beta)}\left[I_{1-}^{\rho}|f(s)|+|\lambda| I_{1-}^{\rho+p} I_{0+}^{q}|g(s)|\right] d s \\
& +\int_{t_{1}}^{t_{2}} \frac{\left|t_{2}-s\right|^{\beta-1}}{\Gamma(\beta)}\left[I_{1-}^{\rho}|f(s)|+|\lambda| I_{1-}^{\rho+p} I_{0+}^{q}|g(s)|\right] d s \mid \\
& +\left|a_{1}\left(t_{2}\right)-a_{1}\left(t_{1}\right)\right|\left\{|\delta| \int_{0}^{\mu} \frac{(\mu-s)^{\beta-1}}{\Gamma(\beta)}\left[I_{1-}^{\rho}|f(s)|+|\lambda| I_{1-}^{\rho+p} I_{0+}^{q}|g(s)|\right] d s\right. \\
& \left.+\int_{0}^{1} \frac{(1-s)^{\beta-1}}{\Gamma(\beta)}\left[I_{1-}^{\rho}|f(s)|+|\lambda| I_{1-}^{\rho+p} I_{0+}^{q}|g(s)|\right] d s\right\} \\
& +\left|a_{2}\left(t_{2}\right)-a_{2}\left(t_{1}\right)\right| \int_{0}^{\xi} \frac{(\xi-s)^{\beta-1}}{\Gamma(\beta)}\left[I_{1-}^{\rho}|f(s)|+|\lambda| I_{1-}^{\rho+p} I_{0+}^{q}|g(s)|\right] d s
\end{aligned}
$$




$$
\begin{aligned}
\leq & {\left[\left|t_{2}^{\beta}-t_{1}^{\beta}\right|+2\left(t_{2}-t_{1}\right)^{\beta}+\frac{(|\delta|+1)}{|\Lambda|}\left(\xi^{\beta+1}\left|t_{2}^{\beta}-t_{1}^{\beta}\right|+\xi^{\beta}\left|t_{1}^{\beta+1}-t_{2}^{\beta+1}\right|\right)\right.} \\
& \left.+\frac{1}{|\Lambda|}\left(\left|1-\delta \mu^{\beta+1}\right|\left|t_{2}^{\beta}-t_{1}^{\beta}\right|+\left|1-\delta \mu^{\beta}\right|\left|t_{1}^{\beta+1}-t_{2}^{\beta+1}\right|\right)\right] \\
& \times\left\{\frac{\theta_{1}(r)}{\Gamma(\beta+1) \Gamma(\rho)} \int_{0}^{1} \sigma_{1}(s) d s+\frac{|\lambda| \theta_{2}(r)}{\Gamma(\beta+1) \Gamma(\rho+p) \Gamma(q)} \int_{0}^{1} \sigma_{2}(s) d s\right\}
\end{aligned}
$$

which tends to zero independently of $y \in \mathscr{B}_{r}$ as $t_{2}-t_{1} \rightarrow 0$. As a consequence of Steps 1-3, Arzelá-Ascoli theorem concludes that $\Omega: C([0,1], \mathbb{R}) \rightarrow \mathscr{P}(C([0,1], \mathbb{R}))$ is completely continuous.

Step 4. ( $\Omega$ is upper semicontinuous).

By [23, Proposition 1.2] (see (iv)), it is sufficient to show that $\Omega$ has a closed graph. Let $y_{n} \rightarrow y_{*}, h_{n} \in \Omega\left(y_{n}\right)$ and $h_{n} \rightarrow h_{*}$. Then we need to show that $h_{*} \in \Omega\left(y_{*}\right)$. For $h_{n} \in \Omega\left(y_{n}\right)$, there exist $f_{n} \in S_{F, y_{n}}, g_{n} \in \widehat{S}_{G, y_{n}}$ such that, for each $t \in[0,1]$,

$$
\begin{aligned}
h_{n}(t)= & \int_{0}^{t} \frac{(t-s)^{\beta-1}}{\Gamma(\beta)}\left[I_{1-}^{\rho} f_{n}(s)-\lambda I_{1-}^{\rho+p} I_{0+}^{q} g_{n}(s)\right] d s \\
& +a_{1}(t)\left\{\delta \int_{0}^{\mu} \frac{(\mu-s)^{\beta-1}}{\Gamma(\beta)}\left[I_{1-}^{\rho} f_{n}(s)-\lambda I_{1-}^{\rho+p} I_{0+}^{q} g_{n}(s)\right] d s\right. \\
& \left.-\int_{0}^{1} \frac{(1-s)^{\beta-1}}{\Gamma(\beta)}\left[I_{1-}^{\rho} f_{n}(s)-\lambda I_{1-}^{\rho+p} I_{0+}^{q} g_{n}(s)\right] d s\right\} \\
& +a_{2}(t) \int_{0}^{\xi} \frac{(\xi-s)^{\beta-1}}{\Gamma(\beta)}\left[I_{1-}^{\rho} f_{n}(s)-\lambda I_{1-}^{\rho+p} I_{0+}^{q} g_{n}(s)\right] d s,
\end{aligned}
$$

Thus it suffices to show that there exist $f_{*} \in S_{F, y_{*}}, g_{*} \in \widehat{S}_{G, y_{*}}$ such that, for each $t \in[0,1]$,

$$
\begin{aligned}
h_{*}(t)= & \int_{0}^{t} \frac{(t-s)^{\beta-1}}{\Gamma(\beta)}\left[I_{1-}^{\rho} f_{*}(s)-\lambda I_{1-}^{\rho+p} I_{0+}^{q} g_{*}(s)\right] d s \\
& +a_{1}(t)\left\{\delta \int_{0}^{\mu} \frac{(\mu-s)^{\beta-1}}{\Gamma(\beta)}\left[I_{1-}^{\rho} f_{*}(s)-\lambda I_{1-}^{\rho+p} I_{0+}^{q} g_{*}(s)\right] d s\right. \\
& \left.-\int_{0}^{1} \frac{(1-s)^{\beta-1}}{\Gamma(\beta)}\left[I_{1-}^{\rho} f_{*}(s)-\lambda I_{1-}^{\rho+p} I_{0+}^{q} g_{*}(s)\right] d s\right\} \\
& +a_{2}(t) \int_{0}^{\xi} \frac{(\xi-s)^{\beta-1}}{\Gamma(\beta)}\left[I_{1-}^{\rho} f_{*}(s)-\lambda I_{1-}^{\rho+p} I_{0+}^{q} g_{*}(s)\right] d s .
\end{aligned}
$$


Let us consider the continuous linear operator $\Theta: L^{1}([0,1], \mathbb{R}) \rightarrow C([0,1], \mathbb{R})$ defined by

$$
\begin{aligned}
(\Theta(f, g))(t)= & \int_{0}^{t} \frac{(t-s)^{\beta-1}}{\Gamma(\beta)}\left[I_{1-}^{\rho} f(s)-\lambda I_{1-}^{\rho+p} I_{0+}^{q} g(s)\right] d s \\
& +a_{1}(t)\left\{\delta \int_{0}^{\mu} \frac{(\mu-s)^{\beta-1}}{\Gamma(\beta)}\left[I_{1-}^{\rho} f(s)-\lambda I_{1-}^{\rho+p} I_{0+}^{q} g(s)\right] d s\right. \\
& \left.-\int_{0}^{1} \frac{(1-s)^{\beta-1}}{\Gamma(\beta)}\left[I_{1-}^{\rho} f(s)-\lambda I_{1-}^{\rho+p} I_{0+}^{q} g(s)\right] d s\right\} \\
& +a_{2}(t) \int_{0}^{\xi} \frac{(\xi-s)^{\beta-1}}{\Gamma(\beta)}\left[I_{1-}^{\rho} f(s)-\lambda I_{1-}^{\rho+p} I_{0+}^{q} g(s)\right] d s .
\end{aligned}
$$

Observe that

$$
\begin{aligned}
& \left\|h_{n}(t)-h_{*}(t)\right\| \\
= & \| \int_{0}^{t} \frac{(t-s)^{\beta-1}}{\Gamma(\beta)}\left[I_{1-}^{\rho}\left(f_{n}(s)-f_{*}(s)\right)-\lambda I_{1-}^{\rho+p} I_{0+}^{q}\left(g_{n}(s)-g_{*}(s)\right)\right] d s \\
& +a_{1}(t)\left\{\delta \int_{0}^{\mu} \frac{(\mu-s)^{\beta-1}}{\Gamma(\beta)}\left[I_{1-}^{\rho}\left(f_{n}(s)-f_{*}(s)\right)-\lambda I_{1-}^{\rho+p} I_{0+}^{q}\left(g_{n}(s)-g_{*}(s)\right)\right] d s\right. \\
& \left.-\int_{0}^{1} \frac{(1-s)^{\beta-1}}{\Gamma(\beta)}\left[I_{1-}^{\rho}\left(f_{n}(s)-f_{*}(s)\right)-\lambda I_{1-}^{\rho+p} I_{0+}^{q}\left(g_{n}(s)-g_{*}(s)\right)\right] d s\right\} \\
& +a_{2}(t) \int_{0}^{\xi} \frac{(\xi-s)^{\beta-1}}{\Gamma(\beta)}\left[I_{1-}^{\rho}\left(f_{n}(s)-f_{*}(s)\right)-\lambda I_{1-}^{\rho+p} I_{0+}^{q}\left(g_{n}(s)-g_{*}(s)\right)\right] d s \| \rightarrow 0 \text { as } n \rightarrow \infty .
\end{aligned}
$$

Thus, it follows by a closed graph operator result [26] that $\Theta \circ S_{B}$ is a closed graph operator, where $S_{B}=S_{F} \cup \widehat{S}_{G}$. Moreover, we have $h_{n}(t) \in \Theta\left(S_{B, y_{n}}\right)$. Since $y_{n} \rightarrow y_{*}, h_{n} \rightarrow h_{*}$, therefore, we have

$$
\begin{aligned}
h_{*}(t)= & \int_{0}^{t} \frac{(t-s)^{\beta-1}}{\Gamma(\beta)}\left[I_{1-}^{\rho} f_{*}(s)-\lambda I_{1-}^{\rho+p} I_{0+}^{q} g_{*}(s)\right] d s \\
& +a_{1}(t)\left\{\delta \int_{0}^{\mu} \frac{(\mu-s)^{\beta-1}}{\Gamma(\beta)}\left[I_{1-}^{\rho} f_{*}(s)-\lambda I_{1-}^{\rho+p} I_{0+}^{q} g_{*}(s)\right] d s\right. \\
& \left.-\int_{0}^{1} \frac{(1-s)^{\beta-1}}{\Gamma(\beta)}\left[I_{1-}^{\rho} f_{*}(s)-\lambda I_{1-}^{\rho+p} I_{0+}^{q} g_{*}(s)\right] d s\right\} \\
& +a_{2}(t) \int_{0}^{\xi} \frac{(\xi-s)^{\beta-1}}{\Gamma(\beta)}\left[I_{1-}^{\rho} f_{*}(s)-\lambda I_{1-}^{\rho+p} I_{0+}^{q} g_{*}(s)\right] d s,
\end{aligned}
$$

for some $f_{*} \in S_{F, y_{*}}, g_{*} \in \widehat{S}_{G, y_{*}}$.

Step 5. (There exists an open set $\mathscr{V} \subseteq C([0.1], \mathbb{R})$ with $x \notin \theta \Omega(y)$ for any $\theta \in(0,1)$ and all $y \in \partial \mathscr{V})$.

Take $\theta \in(0,1), y \in \theta \Omega(y)$ and $t \in[0,1]$. Then there exist $f, g \in L^{1}([0,1], \mathbb{R})$ with $f \in S_{F, y}, g \in$ $\widehat{S}_{G, y}$ such that

$$
|y(t)| \leq\left(\frac{\theta_{1}\left(\|y\|_{\infty}\right)}{\Gamma(\rho)} \int_{0}^{1} \sigma_{1}(t) d t+\frac{|\lambda| \theta_{2}\left(\|y\|_{\infty}\right)}{\Gamma(\rho+p) \Gamma(q)} \int_{0}^{1} \sigma_{2}(t) d t\right) \tilde{\Delta}
$$


for $t \in[0,1]$. Consequently, it follows that

$$
\frac{\Gamma(\rho) \Gamma(\rho+p) \Gamma(q)\|y\|_{\infty}}{\left(\Gamma(\rho+p) \Gamma(q) \theta_{1}\left(\|y\|_{\infty}\right)\left\|\sigma_{1}\right\|_{L^{1}}+|\lambda| \Gamma(\rho) \theta_{2}\left(\|y\|_{\infty}\right)\left\|\sigma_{2}\right\|_{L^{1}}\right) \tilde{\Delta}} \leq 1 .
$$

In view of $\left(\mathbf{H}_{3}\right)$, there exists $M$ such that $\|y\|_{\infty} \neq M$. Let us set

$$
W=\left\{y \in C([0,1], \mathbb{R}):\|y\|_{\infty}<M\right\} .
$$

Note that the operator $\Omega: \bar{W} \rightarrow \mathscr{P}(C([0,1], \mathbb{R}))$ is upper semicontinuous and completely continuous. From the choice of $W$, there is no $y \in \partial W$ such that $y \in \theta \Omega(y)$ for some $\theta \in(0,1)$. Therefore, by nonlinear alternative of Leray-Schauder type [25], we deduce that $\Omega$ has a fixed point $y \in \bar{W}$, which corresponds to a solution of boundary value problem (1.1). The proof is complete

3.2. Generalized contraction case (Nonconvex valued). In this section, we study the existence of solutions for the inclusions boundary value problem (1.1) with the right-hand side being nonconvex set-valued by applying Wegrzyk's fixed point theorem [Let $(X, d)$ be a complete metric space. If $\Phi: X \rightarrow \mathscr{P}_{c l}(X)$ is a generalized contraction, then Fix $\left.\Phi \neq \emptyset[21]\right]$.

Theorem 3.3. Assume that:

$\left(\mathbf{H}_{4}\right) F, G:[0,1] \times \mathbb{R} \rightarrow \mathscr{P}_{c p}(\mathbb{R})$ are such that $F(\cdot, y(t)):[0,1] \rightarrow \mathscr{P}_{c p}(\mathbb{R})$ and $G(\cdot, y(t)):$ $[0,1] \rightarrow \mathscr{P}_{c p}(\mathbb{R})$ are measurable for each $y \in \mathbb{R}$, where $\mathscr{P}_{c p}(\mathbb{R})=\{\mathscr{U} \in \mathscr{P}(\mathbb{R}): \mathscr{U}$ is compact\};

(H5) $H_{d}(F(t, y), F(t, \bar{y})) \leq \kappa_{1}(t) l_{1}(|y-\bar{y}|)$ and $H_{d}(G(t, y), G(t, \bar{y})) \leq \kappa_{2}(t) l_{2}(|y-\bar{y}|)$ for almost all $t \in[0,1]$ and $y, \bar{y} \in \mathbb{R}$ with $\kappa_{i} \in L^{1}\left([0,1], \mathbb{R}^{+}\right) ; i=1,2, d(0, F(t, 0)) \leq \kappa_{1}(t)$ and $d(0, G(t, 0)) \leq \kappa_{2}(t)$ for almost all $t \in[0,1]$, where $l_{1}, l_{2}: \mathbb{R}_{+} \rightarrow \mathbb{R}_{+}$are strictly increasing.

Then (1.1) has at least one solution on $[0,1]$ if $\gamma_{1} l_{1}, \gamma_{2} l_{2}: \mathbb{R}_{+} \rightarrow \mathbb{R}_{+}$are strict comparison functions, where

$$
\gamma_{1}=\frac{\tilde{\Delta}}{\Gamma(\rho)}\left\|\kappa_{1}\right\|_{L^{1}}, \gamma_{2}=\frac{|\lambda| \tilde{\Delta}}{\Gamma(\rho+p) \Gamma(q)}\left\|\kappa_{2}\right\|_{L^{1}}
$$

and $\tilde{\Delta}$ is defined by (3.1).

Proof. Let us consider the operator $\Omega: C([0,1], \mathbb{R}) \rightarrow \mathscr{P}(C([0,1], \mathbb{R}))$ defined in Theorem 3.2. Suppose that $\gamma_{1} l_{1}, \gamma_{2} l_{2}: \mathbb{R}_{+} \rightarrow \mathbb{R}_{+}$are strict comparison functions. It follows by assumptions $\left(\mathbf{H}_{4}\right)$ and $\left(\mathbf{H}_{5}\right)$ that $F(\cdot, y(\cdot))$ and $G(\cdot, y(\cdot))$ are measurable and have measurable selections $v(\cdot)$ and $z(\cdot)$ (see [27], Theorem III.6). Also, $\kappa_{i} \in L^{1}([0,1], \mathbb{R}) ; i=1,2$, and

$$
\begin{aligned}
|v(t)| & \leq d(0, F(t, 0))+H_{d}(F(t, 0), F(t, y(t)) \\
& \leq \kappa_{1}(t)+\kappa_{1}(t) l_{1}(|y(t)|) \\
& \leq\left(1+l_{1}\left(\|y\|_{\infty}\right)\right) \kappa_{1}(t) .
\end{aligned}
$$

Similarly, we can find that

$$
|z(t)| \leq\left(1+l_{2}\left(\|y\|_{\infty}\right)\right) \kappa_{2}(t) .
$$

Thus the sets $S_{F, y}$ and $\widehat{S}_{G, y}$ are nonempty for each $y \in C([0,1], \mathbb{R})$. Now we need to show that the operator $\Omega$ satisfies the assumptions of Wegrzyk's fixed point theorem [21]. First, we show that $\Omega(y) \in \mathscr{P}_{c l}(C([0,1], \mathbb{R}))$ for each $y \in C([0,1], \mathbb{R})$. Let $\left\{u_{n}\right\}_{n \geq 0} \in \Omega(y)$ be a convergent 
sequence such that $u_{n} \rightarrow u(n \rightarrow \infty)$ in $C([0,1], \mathbb{R})$. Then $u \in C([0,1], \mathbb{R})$ and there exist $v_{n} \in S_{F, y}$ and $z_{n} \in \widehat{S}_{G, y}$ such that, for each $t \in[0,1]$,

$$
\begin{aligned}
u_{n}(t)= & \int_{0}^{t} \frac{(t-s)^{\beta-1}}{\Gamma(\beta)}\left[I_{1-}^{\rho} v_{n}(s)-\lambda I_{1-}^{\rho+p} I_{0+}^{q} z_{n}(s)\right] d s \\
& +a_{1}(t)\left\{\delta \int_{0}^{\mu} \frac{(\mu-s)^{\beta-1}}{\Gamma(\beta)}\left[I_{1-}^{\rho} v_{n}(s)-\lambda I_{1-}^{\rho+p} I_{0+}^{q} z_{n}(s)\right] d s\right. \\
& \left.-\int_{0}^{1} \frac{(1-s)^{\beta-1}}{\Gamma(\beta)}\left[I_{1-}^{\rho} v_{n}(s)-\lambda I_{1-}^{\rho+p} I_{0+}^{q} z_{n}(s)\right] d s\right\} \\
& +a_{2}(t) \int_{0}^{\xi} \frac{(\xi-s)^{\beta-1}}{\Gamma(\beta)}\left[I_{1-}^{\rho} v_{n}(s)-\lambda I_{1-}^{\rho+p} I_{0+}^{q} z_{n}(s)\right] d s .
\end{aligned}
$$

By compactness of $F$ and $G$, the sequences $\left\{v_{n}\right\}_{n \geq 1}$ and $\left\{z_{n}\right\}_{n \geq 1}$ have subsequences, which converge to some $v, z \in L^{1}([0,1], \mathbb{R})$. Thus, $v \in S_{F, y}, z \in \widehat{S}_{G, y}$ and for each $t \in[0,1]$, we obtain

$$
\begin{aligned}
u_{n}(t) \rightarrow u(t)= & \int_{0}^{t} \frac{(t-s)^{\beta-1}}{\Gamma(\beta)}\left[I_{1-}^{\rho} v(s)-\lambda I_{1-}^{\rho+p} I_{0+}^{q} z(s)\right] d s \\
& +a_{1}(t)\left[\delta \int_{0}^{\mu} \frac{(\mu-s)^{\beta-1}}{\Gamma(\beta)}\left[I_{1-}^{\rho} v(s)-\lambda I_{1-}^{\rho+p} I_{0+}^{q} z(s)\right] d s\right. \\
& \left.-\int_{0}^{1} \frac{(1-s)^{\beta-1}}{\Gamma(\beta)}\left[I_{1-}^{\rho} v(s)-\lambda I_{1-}^{\rho+p} I_{0+}^{q} z(s)\right] d s\right] \\
& +a_{2}(t) \int_{0}^{\xi} \frac{(\xi-s)^{\beta-1}}{\Gamma(\beta)}\left[I_{1-}^{\rho} v(s)-\lambda I_{1-}^{\rho+p} I_{0+}^{q} z(s)\right] d s .
\end{aligned}
$$

Hence $u \in \Omega(y)$ and so $\Omega$ is closed-valued.

Next, we establish that

$$
H_{d}(\Omega(y), \Omega(\bar{y})) \leq \gamma_{1} l_{1}\left(\|y-\bar{y}\|_{\infty}\right)+\gamma_{2} l_{2}\left(\|y-\bar{y}\|_{\infty}\right) \text { for each } y, \bar{y} \in C([0,1], \mathbb{R}) .
$$

Let $y, \bar{y} \in C([0,1], \mathbb{R})$ and $h_{1} \in \Omega(y)$. Then there exist $v_{1}(t) \in S_{F, y}$ and $z_{1}(t) \in \widehat{S}_{G, y}$ such that, for each $t \in[0,1]$,

$$
\begin{aligned}
h_{1}(t)= & \int_{0}^{t} \frac{(t-s)^{\beta-1}}{\Gamma(\beta)}\left[I_{1-}^{\rho} v_{1}(s)-\lambda I_{1-}^{\rho+p} I_{0+}^{q} z_{1}(s)\right] d s \\
& +a_{1}(t)\left\{\delta \int_{0}^{\mu} \frac{(\mu-s)^{\beta-1}}{\Gamma(\beta)}\left[I_{1-}^{\rho} v_{1}(s)-\lambda I_{1-}^{\rho+p} I_{0+}^{q} z_{1}(s)\right] d s\right. \\
& \left.-\int_{0}^{1} \frac{(1-s)^{\beta-1}}{\Gamma(\beta)}\left[I_{1-}^{\rho} v_{1}(s)-\lambda I_{1-}^{\rho+p} I_{0+}^{q} z_{1}(s)\right] d s\right\} \\
& +a_{2}(t) \int_{0}^{\xi} \frac{(\xi-s)^{\beta-1}}{\Gamma(\beta)}\left[I_{1-}^{\rho} v_{1}(s)-\lambda I_{1-}^{\rho+p} I_{0+}^{q} z_{1}(s)\right] d s .
\end{aligned}
$$

In view of $\left(\mathbf{H}_{5}\right)$, we have

$$
\begin{aligned}
& H_{d}(F(t, y), F(t, \bar{y})) \leq \kappa_{1}(t) l_{1}(|y-\bar{y}|), \\
& H_{d}(G(t, y), G(t, \bar{y})) \leq \kappa_{2}(t) l_{2}(|y-\bar{y}|) .
\end{aligned}
$$


So, there exist $w \in F(t, \bar{y})$ and $m \in G(t, \bar{y})$ such that

$$
\begin{gathered}
\left|v_{1}(t)-w\right| \leq \kappa_{1}(t) l_{1}(|y(t)-\bar{y}(t)|), t \in[0,1], \\
\left|z_{1}(t)-m\right| \leq \kappa_{2}(t) l_{2}(|y(t)-\bar{y}(t)|), t \in[0,1] .
\end{gathered}
$$

Define $U_{1}, U_{2}:[0,1] \rightarrow \mathscr{P}(\mathbb{R})$ by

$$
\begin{aligned}
& U_{1}(t)=\left\{w \in \mathbb{R}:\left|v_{1}(t)-w\right| \leq \kappa_{1}(t) l_{1}(|y(t)-\bar{y}(t)|)\right\}, \\
& U_{2}(t)=\left\{m \in \mathbb{R}:\left|z_{1}(t)-v\right| \leq \kappa_{2}(t) l_{2}(|y(t)-\bar{y}(t)|)\right\} .
\end{aligned}
$$

Since the nonempty closed set-valued operators $U_{1}(t) \cap F(t, \bar{y})$ and $U_{2}(t) \cap G(t, \bar{y})$ are measurable (Proposition III.4 [27]), there exist functions $v_{2}(t), z_{2}(t)$ which are measurable selection for $U_{1}(t) \cap F(t, \bar{y})$ and $U_{2}(t) \cap G(t, \bar{y})$ respectively. So $v_{2}(t) \in F(t, \bar{y}), z_{2}(t) \in G(t, \bar{y})$ and for each $t \in$ $[0,1]$, we have $\left|v_{1}(t)-v_{2}(t)\right| \leq \kappa_{1}(t) l_{1}(|y(t)-\bar{y}(t)|)$ and $\left|z_{1}(t)-z_{2}(t)\right| \leq \kappa_{2}(t) l_{2}(|y(t)-\bar{y}(t)|)$. For each $t \in[0,1]$, let us define

$$
\begin{aligned}
h_{2}(t)= & \int_{0}^{t} \frac{(t-s)^{\beta-1}}{\Gamma(\beta)}\left[I_{1-}^{\rho} v_{2}(s)-\lambda I_{1-}^{\rho+p} I_{0+}^{q} z_{2}(s)\right] d s \\
& +a_{1}(t)\left\{\delta \int_{0}^{\mu} \frac{(\mu-s)^{\beta-1}}{\Gamma(\beta)}\left[I_{1-}^{\rho} v_{2}(s)-\lambda I_{1-}^{\rho+p} I_{0+}^{q} z_{2}(s)\right] d s\right. \\
& \left.-\int_{0}^{1} \frac{(1-s)^{\beta-1}}{\Gamma(\beta)}\left[I_{1-}^{\rho} v_{2}(s)-\lambda I_{1-}^{\rho+p} I_{0+}^{q} z_{2}(s)\right] d s\right\} \\
& +a_{2}(t) \int_{0}^{\xi} \frac{(\xi-s)^{\beta-1}}{\Gamma(\beta)}\left[I_{1-}^{\rho} v_{2}(s)-\lambda I_{1-}^{\rho+p} I_{0+}^{q} z_{2}(s)\right] d s .
\end{aligned}
$$

Thus

$$
\begin{aligned}
& \left|h_{1}(t)-h_{2}(t)\right| \\
\leq & \int_{0}^{t} \frac{(t-s)^{\beta-1}}{\Gamma(\beta)}\left[I_{1-}^{\rho}\left|v_{1}(s)-v_{2}(s)\right|+|\lambda| I_{1-}^{\rho+p} I_{0+}^{q}\left|z_{1}(s)-z_{2}(s)\right|\right] d s \\
& +\left|a_{1}(t)\right|\left\{|\delta| \int_{0}^{\mu} \frac{(\mu-s)^{\beta-1}}{\Gamma(\beta)}\left[I_{1-}^{\rho}\left|v_{1}(s)-v_{2}(s)\right|+|\lambda| I_{1-}^{\rho+p} I_{0+}^{q}\left|z_{1}(s)-z_{2}(s)\right|\right] d s\right. \\
& \left.+\int_{0}^{1} \frac{(1-s)^{\beta-1}}{\Gamma(\beta)}\left[I_{1-}^{\rho}\left|v_{1}(s)-v_{2}(s)\right|+|\lambda| I_{1-}^{\rho+p} I_{0+}^{q}\left|z_{1}(s)-z_{2}(s)\right|\right] d s\right\} \\
& +\left|a_{2}(t)\right| \int_{0}^{\xi} \frac{(\xi-s)^{\beta-1}}{\Gamma(\beta)}\left[I_{1-}^{\rho}\left|v_{1}(s)-v_{2}(s)\right|+|\lambda| I_{1-}^{\rho+p} I_{0+}^{q}\left|z_{1}(s)-z_{2}(s)\right|\right] d s \\
\leq & \left(\frac{1}{\Gamma(\rho)} \int_{0}^{1} \kappa_{1}(s) l_{1}\left(\|y-\bar{y}\|_{\infty}\right) d s+\frac{|\lambda|}{\Gamma(\rho+p) \Gamma(q)} \int_{0}^{1} \kappa_{2}(s) l_{2}\left(\|y-\bar{y}\|_{\infty}\right) d s\right) \tilde{\Delta},
\end{aligned}
$$

which implies that

$$
\left\|h_{1}-h_{2}\right\|_{\infty} \leq \frac{\left\|\kappa_{1}\right\|_{L^{1}} \tilde{\Delta}}{\Gamma(\rho)} l_{1}\left(\|y-\bar{y}\|_{\infty}\right)+\frac{|\lambda|\left\|\kappa_{2}\right\|_{L^{1}} \tilde{\Delta}}{\Gamma(\rho+p) \Gamma(q)} l_{2}\left(\|y-\bar{y}\|_{\infty}\right),
$$

where $\tilde{\Delta}$ is defined by (3.1). Analogously, interchanging the roles of $y$ and $\bar{y}$, we can obtain

$$
H_{d}(\Omega(y), \Omega(\bar{y})) \leq \gamma_{1} l_{1}\left(\|y-\bar{y}\|_{\infty}\right)+\gamma_{2} l_{2}\left(\|y-\bar{y}\|_{\infty}\right) .
$$


Therefore, $\Omega$ is a generalized contraction. Thus, it follows by Wegrzyk's fixed point theorem [21] that $\Omega$ has a fixed point $y$, which is a solution of (1.1). This completes the proof.

3.3. Examples. Consider the following multivalued boundary value problem

$$
\left\{\begin{array}{l}
{ }^{C} \mathscr{D}_{1-}^{5 / 4}{ }^{R L} \mathscr{D}_{0+}^{1 / 3} y(t) \in F(t, y(t))-1 / 8 I_{1-}^{3 / 2} I_{0+}^{5 / 2} G(t, y(t)), t \in[0,1], \\
y(0)=y(1 / 5)=0, \quad y(1)=1 / 10 y(2 / 5), 0<\xi<\mu<1,
\end{array}\right.
$$

where $\rho=5 / 4, \beta=1 / 3, \lambda=1 / 8, p=3 / 2, q=5 / 2, \xi=1 / 5, \delta=1 / 10, \mu=2 / 5$. With the given data, we find that $|\Lambda| \approx 0.459225099353931$. Moreover,

$$
\begin{aligned}
& \bar{a}_{1}=\max _{t \in[0,1]}\left|a_{1}(t)\right|=\left|a_{1}(t)\right|_{t=1} \approx 1.018765826981695, \\
& \bar{a}_{2}=\max _{t \in[0,1]}\left|a_{2}(t)\right|=\left|a_{2}(t)\right|_{t=t_{a_{2}}} \approx 1.014158908373613,
\end{aligned}
$$

where

$$
t_{a_{2}}=\frac{\beta\left(1-\delta \mu^{\beta+1}\right)}{\left(1-\delta \mu^{\beta}\right)(\beta+1)} \approx 0.261931192257803<1 .
$$

Consequently, we get

$$
\tilde{\Delta}=\frac{1}{\Gamma(\beta+1)}\left[1+\bar{a}_{1}(|\delta|+1)+\bar{a}_{2}\right] \approx 3.510496352312309 .
$$

(a) For illustrating Theorem 3.2, we choose $F$ and $G$ in (3.4) as follows:

$$
F(t, y)=\left[\frac{y|y|}{4(1+|y|)}, \frac{e^{-t}}{10} \sin y\right], G(t, y)=\left[\frac{e^{-t}}{2} \tan ^{-1} y, \frac{1}{2\left(t^{2}+1\right)} \cos y\right],
$$

and observe that

$$
\|F(t, y)\|_{\mathscr{P}}:=\sup \{|v|, v \in F(t, y)\} \leq(1 / 4)|y|,\|G(t, y)\|_{\mathscr{P}}:=\sup \{|u|, u \in G(t, y)\} \leq \pi / 4 \text {. }
$$

With $\sigma_{1}(t)=1, \theta_{1}(|y|)=|y| / 4, \sigma_{2}(t)=1, \theta_{1}(|y|)=\pi / 4$, we note that the condition $\left(H_{3}\right)$ holds true for $M>5.076959728977279$. Clearly, all the conditions of Theorem 3.2 are satisfied and hence its conclusion applies to the problem (3.4) with $F, G$ given by (3.5).

(b) In order to explain Theorem 3.3 (nonconvex-valued case), we take $F, G:[0,1] \times \mathbb{R} \rightarrow \mathscr{P}(\mathbb{R})$ in (3.4) as given by

$$
F(t, y)=\left[\frac{1}{6}, \frac{t+1 / 2}{1+t^{2}} \tan ^{-1} y+\frac{1}{2}\right], G(t, y)=\left[\frac{1}{10}, \frac{y}{\left(3+t^{2}\right)}+\frac{1}{5}\right] .
$$

Notice that

$$
\begin{gathered}
\sup \{|y|, y \in F(t, y)\} \leq \frac{(t+1 / 2) \pi}{2\left(1+t^{2}\right)}+\frac{1}{2}, \sup \{|z|, z \in G(t, y)\} \leq \frac{1}{\left(3+t^{2}\right)}+1 / 5, \\
H_{d}(F(t, y), F(t, \bar{y})) \leq \frac{(t+1 / 2)}{1+t^{2}}(|y-\bar{y}|), H_{d}(G(t, y), G(t, \bar{y})) \leq \frac{1}{\left(3+t^{2}\right)}(|y-\bar{y}|) .
\end{gathered}
$$

Fixing $\kappa_{1}(t)=\frac{(t+1 / 2)}{1+t^{2}}, \kappa_{2}(t)=\frac{1}{\left(3+t^{2}\right)}$, we observe that $d(0, F(t, 0)) \leq \kappa_{1}(t)$ and $d(0, G(t, 0)) \leq$ $\kappa_{2}(t)$ for almost all $t \in[0,1]$. Using $\left\|\kappa_{1}\right\|_{L^{1}}=\frac{1}{2} \ln 2+\pi / 8,\left\|\kappa_{2}\right\|_{L^{1}}=\frac{\pi}{6 \sqrt{3}}$, we find that $\gamma_{1}=\frac{\tilde{\Delta}}{\Gamma(\rho)}\left\|\kappa_{1}\right\|_{L^{1}} \approx 2.863202698624739, \gamma_{2}=\frac{|\lambda| \tilde{\Delta}}{\Gamma(\rho+p) \Gamma(q)}\left\|\kappa_{2}\right\|_{L^{1}} \approx 0.062043633931219$. 
Letting $l_{1}(y)=l_{2}(y)=y$, we have

$$
H_{d}(F(t, y), F(t, \bar{y})) \leq \kappa_{1}(t)(|y-\bar{y}|), \text { and } H_{d}(G(t, y), G(t, \bar{y})) \leq \kappa_{2}(t)(|y-\bar{y}|) .
$$

Clearly all the conditions of Theorem 3.3 are satisfied. Hence, the problem (3.4) with $F$ and $G$ given by (3.6) has at least one solutions on $[0,1]$.

\section{THE DIMENSION OF THE SOLUTION}

In this section, we are concerned with the dimension of the solution set for problem (1.1). The following lemmas are the main tools of our study.

Lemma 4.1. (Dzedzej and Gelman [25]) Let $F:[0, \rho] \rightarrow \mathscr{P}_{c, c p}(\mathbb{R})$ be a measurable map such that the Lebesgue measure $\mu$ of the set $\{t: \operatorname{dim} F(t)<1\}$ is zero, where $\mathscr{P}_{c, c p}(\mathbb{R})=\{\mathscr{U} \in$ $\mathscr{P}(\mathbb{R}): \mathscr{U}$ is convex and compact $\}$. Then there are arbitrarily many linearly independent measurable selections $x_{1}(\cdot), x_{2}(\cdot), \ldots, x_{m}(\cdot)$ of $F$.

Lemma 4.2. ([28]) Let $C$ be a nonempty closed convex subset of a Banach space $X$. Suppose that $\gamma$ is a $\varphi$-measure of noncompactness on $C$ and $F: C \rightarrow \mathscr{P}_{b, c, c l}(C)$ is a continuous $(\gamma, \varphi)$ condensing map, where $\mathscr{P}_{b, c, c l}(C)=\{\mathscr{U} \in \mathscr{P}(C): \mathscr{U}$ is bounded, convex and closed $\}$. If $\operatorname{dim} F(x) \geq n$ for each $x \in C$, then $\operatorname{dim} F i x(F) \geq n$.

Let $S([0, \bar{\rho}])$ denote the set of all solutions of $(1.1)$ on the interval $[0, \bar{\rho}]$, where $0<\bar{\rho} \leq 1$.

Theorem 4.3. Let $F, G:[0, \bar{\rho}] \times \mathbb{R} \rightarrow \mathscr{P}_{c p, c}(\mathbb{R})$ satisfy $\left(\mathbf{H}_{\mathbf{4}}\right)$ and $\left(\mathbf{H}_{\mathbf{5}}\right)$ and suppose that the Lebesgue measures $\mu_{1}, \mu_{2}$ of the sets $\{t: \operatorname{dim} F(t, y)<1\}$ and $\{t: \operatorname{dim} G(t, y)<1\}$ respectively are zero for some $y \in \mathbb{R}$. Then, for each $\bar{\rho}, 0<\bar{\rho}<1$, the set $S([0, \bar{\rho}])$ of solutions for the problem (1.1) has an infinite dimension.

Proof. Define the operator $\Omega$ by

$$
\begin{aligned}
\Omega(y)=\{h \in C([0, \bar{\rho}], \mathbb{R}): \quad & h(t)=\int_{0}^{t} \frac{(t-s)^{\beta-1}}{\Gamma(\beta)}\left[I_{1-}^{\rho} f(s)-\lambda I_{1-}^{\rho+p} I_{0+}^{q} g(s)\right] d s \\
& +a_{1}(t)\left[\delta \int_{0}^{\mu} \frac{(\mu-s)^{\beta-1}}{\Gamma(\beta)}\left[I_{1-}^{\rho} f(s)-\lambda I_{1-}^{\rho+p} I_{0+}^{q} g(s)\right] d s\right. \\
& \left.-\int_{0}^{1} \frac{(1-s)^{\beta-1}}{\Gamma(\beta)}\left[I_{1-}^{\rho} f(s)-\lambda I_{1-}^{\rho+p} I_{0+}^{q} g(s)\right] d s\right] \\
& \left.+a_{2}(t) \int_{0}^{\xi} \frac{(\xi-s)^{\beta-1}}{\Gamma(\beta)}\left[I_{1-}^{\rho} f(s)-\lambda I_{1-}^{\rho+p} I_{0+}^{q} g(s)\right] d s\right\},
\end{aligned}
$$

for $f \in S_{F, y}, g \in \widehat{S}_{G, y}$. Then, as in the proof of Theorem 3.2, $\Omega(y) \in \mathscr{P}_{c p, c}(C([0, \bar{\rho}], \mathbb{R}))$ for each $y \in C([0, \bar{\rho}], \mathbb{R})$ and it is a generalized contraction by Theorem 3.3. We next show that $\operatorname{dim} \Omega(y) \geq m$ for any $y \in C([0, \bar{\rho}], \mathbb{R})$ and arbitrary $m \in \mathbb{N}$. Consider $W(t)=F(t, y(t))$ and $Z(t)=G(t, y(t))$. By Lemma 4.1, there exist linearly independent measurable selections $u_{1}(\cdot)$, 
$u_{2}(\cdot), \ldots, u_{m}(\cdot)$ of $W$ and $v_{1}(\cdot), v_{2}(\cdot), \ldots, v_{m}(\cdot)$ of $Z$. Set

$$
\begin{aligned}
y_{i}(t)= & \int_{0}^{t} \frac{(t-s)^{\beta-1}}{\Gamma(\beta)}\left[I_{1-}^{\rho} u_{i}(s)-\lambda I_{1-}^{\rho+p} I_{0+}^{q} v_{i}(s)\right] d s \\
& +a_{1}(t)\left\{\delta \int_{0}^{\mu} \frac{(\mu-s)^{\beta-1}}{\Gamma(\beta)}\left[I_{1-}^{\rho} u_{i}(s)-\lambda I_{1-}^{\rho+p} I_{0+}^{q} v_{i}(s)\right] d s\right. \\
& \left.-\int_{0}^{1} \frac{(1-s)^{\beta-1}}{\Gamma(\beta)}\left[I_{1-}^{\rho} u_{i}(s)-\lambda I_{1-}^{\rho+p} I_{0+}^{q} v_{i}(s)\right] d s\right\} \\
& +a_{2}(t) \int_{0}^{\xi} \frac{(\xi-s)^{\beta-1}}{\Gamma(\beta)}\left[I_{1-}^{\rho} u_{i}(s)-\lambda I_{1-}^{\rho+p} I_{0+}^{q} v_{i}(s)\right] d s .
\end{aligned}
$$

Assume that $\sum_{i=1}^{m} a_{i} y_{i}=0$ a.e. in $[0, \bar{\rho}]$. Taking the right-Caputo and left-Riemann-Liouville fractional derivatives respectively a.e. in $[0, \bar{\rho}]$, we have $\sum_{i=1}^{m} a_{i} u_{i}(t)-\lambda I_{1-}^{p} I_{0+}^{q} \sum_{i=1}^{m} a_{i} v_{i}(t)=0$ a.e. in $[0, \bar{\rho}]$ and hence $a_{i}=0$ for all $i$. As a result, $y_{i}(\cdot)$ are linearly independent. Thus $\Omega(y)$ contains an m-dimensional simplex. So $\operatorname{dim} \Omega(y) \geq m$ and it follows by Lemma 4.2 that $\operatorname{Fix}(\Omega)=S([0, \bar{\rho}])$ is infinite dimensional. The proof is complete.

\section{THE CONCLUSION}

We investigated the existence of solutions for a nonlocal multi-valued problem involving right-sided Caputo and left-sided Riemann-Liouville fractional differential operators of different orders and mixed Riemann-Liouville fractional integral operators. In our first result, we applied the nonlinear alternative of Leray-Schauder type to obtain the existence of solutions for the problem (1.1) with convex-valued maps in the right-hand-side of the inclusions. Then we considered the case of nonconvex multi-valued maps in (1.1) and used Wegrzyk's fixed point theorem to derive an existence result. Finally, the dimension of the solution set for (1.1) was achieved. It is imperative to note that our results correspond to a three-point nonlocal multivalued analogue of (1.1) once we fix $\delta=0$ in the obtained results. Moreover, by taking $\lambda=0$, we obtained the results for the inclusions of the form: ${ }^{C} \mathscr{D}_{1-}^{\rho} R L \mathscr{D}_{0+}^{\beta} y(t) \in F(t, y(t))$ subject to the four-point nonlocal boundary conditions $y(0)=y(\xi)=0, y(1)=\delta y(\mu), 0<\mu<1$. It is worthwhile to point out that the results presented in this paper are new and significantly contribute to the existing literature on this topic.

\section{Acknowledgements}

We thank the referee for his/her constructive remarks on our work.

\section{REFERENCES}

[1] N. Ahmed, D. Vieru, C. Fetecau, N.A. Shah, Convective flows of generalized time-nonlocal nanofluids through a vertical rectangular channel, Phys. Fluids 30 (2018), 052002.

[2] V.V. Tarasova, V.E. Tarasov, Logistic map with memory from economic model, Chaos Solitons Fractals 95 (2017), 84-91.

[3] M. Di Paola, F.P. Pinnola, M. Zingales, Fractional differential equations and related exact mechanical models, Comput. Math. Appl. 66 (2013), 608-620.

[4] L. Peng, Y. Zhou, Bifurcation from interval and positive solutions of the three-point boundary value problem for fractional differential equations, Appl. Math. Comput. 257 (2015), 458-466. 
[5] B. Ahmad, A. Alsaedi, S.K. Ntouyas, J. Tariboon, Hadamard-Type Fractional Differential Equations, Inclusions and Inequalities, Springer, Cham, Switzerland, 2017.

[6] Y. Cui, W. Ma, Q. Sun, X. Su, New uniqueness results for boundary value problem of fractional differential equation, Nonlinear Anal. Model. Control 23 (2018), 31-39.

[7] A. Guezane Lakoud, R. Khaldi, A. Kilicman, Existence of solutions for a mixed fractional boundary value problem, Adv. Difference Equ. 2017 (2017), 164.

[8] B. Ahmad, S.K. Ntouyas, A. Alsaedi, Existence theory for nonlocal boundary value problems involving mixed fractional derivatives, Nonlinear Anal. Model. Control 24 (2019), 937-957.

[9] T.M. Atanackovic, B. Stankovic, On a differential equation with left and right fractional derivatives, Fract. Calc. Appl. Anal. 10 (2007), 139-150.

[10] A.M. Yang, Y. Han, Y.Z. Zhang, L.T. Wang, D. Zhang, X.J. Yang, On nonlocal fractional Volterra integrodifferential equations in fractional steady heat transfer, Thermal Science, 20 (2016), S789-S793.

[11] V. E. Tarasov, Fractional integro-differential equations for electromagnetic waves in dielectric media, Theoretical Math. Phy. 158 (2009), 355-359.

[12] M. Korda, D. Henrion, C.N. Jones, Convex computation of the maximum controlled invariant set for polynomial control systems, SIAM J. Control Optim. 52 (2014), 2944-2969.

[13] J. Bastien, Study of a driven and braked wheel using maximal monotone differential inclusions: Applications to the nonlinear dynamics of wheeled vehicle, Arch. Appl. Mech. 84 (2014), 851-880.

[14] M.F. Danca, Synchronization of piecewise continuous systems of fractional order, Nonlinear Dyn. 78 (2014), 2065-2084.

[15] M. Kisielewicz, Differential inclusions and optimal control, Kluwer, Dordrecht, The Netherlands, 1991.

[16] Y. Cheng, R.P. Agarwal, D. O'Regan, Existence and controllability for nonlinear fractional differential inclusions with nonlocal boundary conditions and time-varying delay, Fract. Calc. Appl. Anal. 21 (2018), 960-980.

[17] S. Abbas, M. Benchohra, J.R. Graef, Coupled systems of Hilfer fractional differential inclusions in Banach spaces, Commun. Pure Appl. Anal. 17 (2018), 2479-2493.

[18] B. Ahmad, S.K. Ntouyas, J. Tariboon, On inclusion problems involving Caputo and Hadamard fractional derivatives, Acta Math. Univ. Comenian. (N.S.) 89 (2020), 169-183.

[19] B. Ahmad, A. Broom, A. Alsaedi, S.K. Ntouyas, Nonlinear integro-differential equations involving mixed right and left fractional derivatives and integrals with nonlocal boundary data, Mathematics, 8 (2020), 336.

[20] H. Covitz and S. B. Nadler Jr., Multivalued contraction mappings in generalized metric spaces, Israel J. Math. 8 (1970), 5-11.

[21] R. Wegrzyk, Fixed point theorems for multifunctions and their applications to functional equations, Dissertationes Math. (Rozprawy Mat.) 201 (1982), 28 pages.

[22] A.A. Kilbas, H.M. Srivastava, J.J. Trujillo, Theory and Applications of Fractional Differential Equations, North-Holland Mathematics Studies, 204.; Elsevier Science B.V.: Amsterdam, The Netherlands, 2006.

[23] K. Deimling, Multivalued Differential Equations, De Gruyter: Berlin, Germany, 1992.

[24] T. A. Lazar, A. Petrusel, N. Shahzad, Fixed points for non-self operators and domain invariance theorems, Nonlinear Anal. 70 (2009), 117-125.

[25] J. Dugundji, A. Granas, Fixed Point Theory, Springer-Verlag, New York, 2005.

[26] A. Lasota, Z. Opial, An application of the Kakutani-Ky Fan theorem in the theory of ordinary differential equations, Bull. Acad. Polon. Sci. Ser.Sci. Math. Astronom. Phys. 13 (1965), 781-786.

[27] C. Castaing, M. Valadier, Convex Analysis and Measurable Multifunctions, Lecture Notes in Mathematics 580, Springer-Verlag, Berlin-Heidelberg-New York, 1977.

[28] R.P. Agarwal, B. Ahmad, A. Alsaedi, N. Shahzad, Dimension of the solution set for fractional differential inclusions, J. Nonlinear Convex Anal. 14 (2013), 319-329. 